\title{
Feedforward self-modeling enhances skill acquisition in children learning trampoline skills
}

\section{Diane M. Ste-Marie ${ }^{1}{ }^{*}$, Kelly Vertes ${ }^{1}$, Amanda M. Rymal' ${ }^{1}$ and Rose Martini'}

Psychomotor Learning Laboratory, School of Human Kinetics, University of Ottawa, Ottawa, ON, Canada

School of Rehabilitation Sciences, University of Ottawa, Ottawa, ON, Canada

\section{Edited by:}

Ernst-Joachim Hossner, University of Bern, Switzerland

\section{Reviewed by:}

Suzete Chiviacowsky, Federal

University of Pelotas, Brazil

Christian Dettmers, Kliniken Schmieder

Konstanz, Germany

\section{*Correspondence:}

Diane M. Ste-Marie, School of Human Kinetics, University of Ottawa, Ottawa, ON, Canada K2W 1 A2.

e-mail:dstmarie@uottawa.ca

\begin{abstract}
The purpose of this research was to examine whether children would benefit from a feedforward self-modeling (FSM) video and to explore possible explanatory mechanisms for the potential benefits, using a self-regulation framework. To this end, children were involved in learning two five-skill trampoline routines. For one of the routines, a FSM video was provided during acquisition, whereas only verbal instructions were provided for the alternate routine. The FSM involved editing video footage such that it showed the learner performing the trampoline routine at a higher skill level than their current capability. Analyses of the data showed that while physical performance benefits were observed for the routine that was learned with the FSM video, no differences were obtained in relation to the self-regulatory measures. Thus, the FSM video enhanced motor skill acquisition, but this could not be explained by changes to the varied self-regulatory processes examined.
\end{abstract}

Keywords: observational learning, self-regulation, self-efficacy, goal setting, motivation

\section{INTRODUCTION}

When researchers define observational learning they often describe it as a process of watching others to assist in the learning of varied skills (e.g., Schunk, 1987; Schmidt and Wrisberg, 2008). Such definitions, however, exclude a set of modeling techniques that encourages the use of the self as a model. There are a number of factors that can be raised to contend that observing the self may in fact be an optimal model. From a neurological perspective, Holmes and Calmels (2008) recent neuroscience review of action observation highlighted the importance of behavioral agency, i.e., viewing the self versus other. Based on that review, observing the self is argued to provide a greater functional correspondence in terms of neural activation between action execution and observation than that of viewing another individual.

Theoretically, Bandura's (1986) Social Cognitive Theory also lends support to the statement of the self being an optimal model. Bandura, for example, reported on the importance of modelobserver similarity strengthening the attention and retention processes of observational learning, thus leading to increased learning benefits from the observation process. Moreover, psychological constructs, such as self-efficacy and other self-regulatory processes are triggered by the observation of mastery experiences. Increased belief in one's capability to succeed in a given task and the engagement of self-regulatory processes have been shown to be related to enhanced skill acquisition (Bandura, 1997). Mastery experiences, as described by Bandura, is basically the observation of the self performing a task successfully. Dowrick (1999) has identified observation of the self performing successfully as self-modeling. Further, he defined two sub-classifications within self-modeling; positive self-review and feedforward. Dowrick described the positive self-review technique as "catch me being good and remind myself of it" (p. 25), whereas feedforward modeling is creating an experience that shows the learner performing at a higher skill level yet to be attained, or performing the skill in a more challenging context.

The current research is interested in the use of the feedforward technique for motor skill acquisition; specifically the acquisition of trampoline skills. While there has been research that has shown the benefits of positive self-review modeling for the learning of swimming skills (Dowrick and Dove, 1980; Starek and McCullagh, 1999; Clark and Ste-Marie, 2007), no research to date has examined the possible benefits of a feedforward technique for motor skill acquisition. Moreover, findings have been equivocal in respect of the benefits of self-modeling for motor skill acquisition. For example, the acquisition of the volleyball serve (Ram and McCullagh, 2003), and figure skating jumps (Law and SteMarie, 2005) did not yield physical performance benefits from a positive self-review intervention. Thus, continued research in the area is warranted to better understand those skills that may benefit from such interventions.

A final important feature of the research is the adoption of a self-regulation framework to examine the possible explanatory mechanisms should any benefits from watching a feedforward self-model accrue. Indeed, others have noted that a weakness with previous research has been the lack of a theoretical questioning as to why it is effective (Dowrick, 1999; Starek and McCullagh, 1999). Clark and Ste-Marie (2007) responded to this weakness by adopting Zimmerman's (2000) self-regulation of learning model to examine the benefits of a positive self-review modeling intervention. Similar to Clark and Ste-Marie, we used Zimmerman's framework due to its broader use of other self-regulatory processes and the fact that it is situated within Bandura's (1986) Social Cognitive Theory. Also, Zimmerman's model works within an event approach to self-regulated learning which defines self-regulation as 
occurring within a temporal entity with a discernable beginning and end, thus allowing for clear examination around an event like that of a trampoline routine. The phases surrounding the event are described as the forethought, performance control, and selfreflection phases (Zimmerman, 2000). In brief, the forethought phase involves those self-regulatory processes and beliefs that precede one's actions and enable it to occur, whereas the performance control phase are those that occur during the actual execution of the action. Finally, the self-reflection phase includes a number of self-regulatory processes that are engaged in subsequent to the action. These phases act in a cyclical fashion as the information from prior performance is used to make adjustments to the current efforts and so forth.

Unique to this research is a more extensive examination of self-regulatory processes and beliefs as possible contributions to the potential advantages accrued from feedforward self-modeling (FSM) than that investigated thus far. Clark and Ste-Marie (2007) examined two self-regulatory processes and beliefs from the forethought phase (self-efficacy and intrinsic interest), and one from the self-reflection phase (self-satisfaction). In that research, children who received a positive self-review modeling intervention not only showed greater physical performance benefits compared to those who had a self-observation intervention or no model, they also showed greater intrinsic interest for swimming and were more selfsatisfied; no differences were found for self-efficacy. Our research focuses on a subset of processes and beliefs situated within the forethought phase of Zimmerman's (2000) model. We are most interested in this phase due to Zimmerman's (2008) contention that those who engage in high-quality forethought improve their self-regulatory functioning during subsequent phases, creating an overall enhancement in learning.

Zimmerman (2000) described the forethought phase as consisting of two categories; task analysis and self-motivational beliefs. Within task analysis are goal setting and strategic planning. Strategic planning involves plans of action or tools used to assist with learning a new skill. Thus, the use of the FSM video herein would be a strategy being used by the learners to assist in achieving their goals. We anticipated that using the FSM video would encourage greater use of other strategies, such as imagery or self-instruction. Rymal et al.'s (2010) research with gymnasts showed that using a FSM video in competition was coupled with the use of a variety of other strategic planning activities.

Goal setting has been identified as an essential component of the learning process because it enables learners to assess their learning progress (see Weinberg and Butt, 2005 for review). Two key characteristics of specificity and proximity distinguish goals (Bandura, 1986). Specificity is determined by whether goals are general (e.g., "to do better") or specific (e.g., "to keep my legs together when I twist"). A learner's progress can be more easily observed when goals are specific, as opposed to general, thus leading to greater persistence with the task (Kyllo and Landers, 1995). Proximity is determined by whether goals deal with the final outcome (outcome goals, e.g., "I want to improve the score in my trampoline routine"), or are more short term and part of the process toward a final outcome; such as "Today I want to connect the seat drop and back drop without an extra bounce." Within the context of this research, we asked the learners whether they had any goals for that day, thus removing the characteristic of proximity. We did, however, examine the characteristic of specificity with the hypothesis that a FSM video would result in the learners setting more specific goals as opposed to when no video is provided.

The second classification within the forethought phase, selfmotivational beliefs, includes outcome expectations, goal orientation, self-efficacy, and task interest (Zimmerman, 2000); with our focus on the last two components. Perceived self-efficacy is related to people's beliefs in their capabilities to produce certain attainments (Bandura, 1997). This is an important self-belief because the more competent learners feel about their capabilities the more likely they are to strive to achieve their goal (Locke and Latham, 1990). Further, a highly self-efficacious person is more likely to persist at acquiring a skill even when having difficulty progressing (Feltz et al., 2008). Given Bandura's (1997) statement that mastery experiences are the strongest predictor of self-efficacy, our hypothesis was that the use of a FSM video would lead to higher levels of self-efficacy than when no video is provided.

Task interest within Zimmerman's (2000) model identifies the importance associated with performing a task for its intrinsic properties, rather than external ends, and thus is very much in line with Deci's (1975) notions of intrinsic motivation. Indeed, Zimmerman refers to Deci's work when conceptualizing this component of his model. As such, children's task interest for learning the trampoline routines was examined with an intrinsic motivation questionnaire. Based on Clark and Ste-Marie's (2007) findings, we hypothesized that children would be more intrinsically motivated while learning a trampoline routine that was accompanied with a FSM video than one that was not.

In sum, children were involved in learning two trampoline routines during a summer camp program. For one routine, a FSM video was created and provided to them during the learning of the routine, whereas no video was created for the alternate routine. We hypothesized that children would show better physical performance, greater strategic planning, more specific goals, greater self-efficacy, and more intrinsic motivation for the routine in which they viewed a FSM video as compared to the one which did not receive a modeling intervention.

\section{MATERIALS AND METHODS PARTICIPANTS}

Thirty-one children ( males $=13$, females $=18$ ) with an age range of 7-13 ( $M$ age $=10.2$ years) were recruited from a local trampoline summer camp. All parents provided informed consent, and children assented prior to participation. The research met the ethical standards of the ethics review board of the University with which the authors are affiliated.

\section{MATERIALS}

A Toshiba Satellite laptop computer with a 15 inch screen was used to display all video footage. A Sony video Handycam (model number DCR-HC65/HC85) mounted on a tripod was used for videotaping. FSM videotapes were created using Dartfish Pro Software (version 4.5.1.0) for each participant. The video footage from the pre-test practice trials were used to create these FSM videos. The video camera was set up on a tripod at a $90^{\circ}$ angle to the trampoline, capturing the side view. For each participant, one of the researchers 
qualified in trampoline (former Canadian Novice National champion), examined all of the footage and selected the best performance of each individual trampoline skill to create the five-skill routine. These skills were then edited together to make it appear as though the learner was performing the skills in immediate succession. The FSM video showed the learner performing the skills in real time. New FSM videos were constructed for each intervention session if the child's performance improved. This was done so that the FSM video would always show the most successful behaviors because researchers have argued that updated videos may be a vital component for the effectiveness of self-modeling interventions (Winfrey and Weeks, 1993; Dowrick, 1999).

\section{Physical performance}

Trampoline routines were scored based on a number of execution variables related to form, amplitude, height, traveling (i.e., remaining in a box area of the trampoline), and performing all skills in a consecutive fashion with no extra bounces. Each trampoline routine consisted of five-skills and each skill could obtain a maximal score of 1 , thus the possible range in score was $0-5$. Deductions related to the variables mentioned occurred if the skill was not performed to the optimal level. Two certified trampoline judges, blind to condition, scored the videotapes of the two trampoline five-skill routines.

\section{Goal setting}

Before physical practice began, participants were asked: "Did you set any goals for today?" The researcher emphasized that there was no right or wrong answer. If a participant had a goal, the researcher asked him/her to verbalize it, and it was recorded on paper.

\section{Strategic planning}

To date, no research in the motor domain using Zimmerman's (2000) self-regulation model has investigated strategic planning. Therefore, the Strategic Planning Questionnaire was created. Of importance in assessing this process is ascertaining the type of strategies that are being used by the learner. Ideas for items on the questionnaire came from Zimmerman's readings on what learners use as strategies to assist in their own learning, as well as ideas from the researchers' own experiences with the learning of trampoline and gymnastic skills. The wording of each item was constructed to be appropriate for children. This questionnaire consisted of 10 questions that participants rated on a five-point Likert scale, ranging from never to always. Examples of items include (1) I blocked out distractions, and (2) I imagined the routine in my head. The other strategies included in the questionnaire involved the use of key words, positive thoughts, controlling affect/negative thoughts, focus on routine, and remembering cues given by coach. The instructions reminded the participant to think about what they did before the routine was practiced and to rate each item based on that time period. The dependent variable was the average score across the 10 statements, yielding a possible range of scores from 0 (never using any of the strategies) to 5 (using every strategy all the time).

Although we did not do a complete full validity and reliability assessment of the questionnaire, certain steps were conducted to ensure that the questionnaire would provide us with information about strategies used by the participants. Validity refers to the degree to which a questionnaire measures what it is purported to measure (Urbina, 2004). Construct validity examines whether a questionnaire measures factors which are theorized to be a part of the psychological processes being measured (Murphy and Davidshofer, 2005). Thus, a team of four researchers, well informed on Zimmerman's (2000) model of self-regulated learning, evaluated whether the Strategic Planning Questionnaire items reflected Zimmerman's concept of strategic planning. The researchers agreed that the items were an accurate representation of those strategies. Face validity is the assessment of whether or not a questionnaire, on the surface, measures the construct (Urbina, 2004). To assess the face validity of the Strategic Planning Questionnaire, 13 trampolinists aged 9-15 and two competitive trampoline coaches were asked to read each item and reflect on whether the item would be used as a strategy by trampolinists when attempting to learn trampoline routines. They were asked to rate their level of agreement on a five-point Likert scale, with one anchored as "not at all," three as "somewhat," and five as "extremely." All questions received ratings of four or above, indicating face validity of the questionnaire.

\section{MOSS-challenge sub-scale}

The Motivation Orientation in Sport Scale (MOSS, Weiss et al., 1985) assessed the children's intrinsic/extrinsic motivation. This scale was chosen because Weiss et al. designed it specifically for children. The scale contains five motivation sub-scales, and we used the challenge sub-scale because of its focus on intrinsic and extrinsic motivation. Weiss et al. reported that the challenge sub-scale demonstrated acceptable internal consistency with a Cronbach's alpha of 0.81 . This sub-scale consists of five items that assesses children's preferences for challenging skills versus easy skills. Each of the five items in the sub-scale contains two statements that can be modified to be consistent with the chosen task. For example, one item consisted of the following two statements, "Some kids like hard trampoline skills because they are a challenge BUT other kids prefer easy trampoline skills that they are sure they can do." Associated with each statement is the valence of "sort of true" or "really true." The items were scored on a four-point ordinal scale with the anchorings 1 (extrinsic) to 4 (intrinsic). The total score for the MOSS-challenge sub-scale was determined by summing the scores given for each item, creating a range from 5 (extrinsic) to 20 (intrinsic).

\section{Self-efficacy}

The self-efficacy measure was developed according to Bandura's (2006) guidelines. The instructions explained that the participants were to evaluate their beliefs in their ability to obtain a specific score on the routine about to be attempted. Nine statements progressed from easily attainable scores, beginning at 1 and increasing incrementally by 0.5 , to the highest score of 5 . For example, the first statement was "I can complete the routine with a score of at least 1," whereas the last statement said "with a score of 5." A description concerning the required performance level for such a score was provided. The participants rated their degree of confidence by recording a number from 0 to 100 on a blank line next to each statement. A Likert scale from 1 to 100, in increments of 10, with the anchors as "cannot do at all" and "really sure I can do," and the 
statement, "sort of can do" at 50 was provided at the top of the questionnaire. Using their recorded scores, an average score of all the items was calculated to represent the participants self-efficacy. Examining the pattern of the responses for all participants showed that they responded in a manner that suggested the questionnaire was understood and completed correctly. Specifically, self-efficacy scores were very high on the first items and gradually decreased as the gradations in challenge increased on the scale.

\section{PROCEDURE}

The experiment was completed across 9 days and involved three different phases: pre-test, acquisition, and retention. All participants were tested individually.

\section{Pre-test phase}

The pre-test consisted of two testing days; 1 day was for the routine that would receive a FSM video (FSM) and another day for the routine which would not receive a video (control). When participants signed up for the research, they were already assigned to a specific level based on their current ability determined by the trampoline club. Each level encompassed a set of progressions that the athlete had to achieve to move up to the next level. For the purpose of this research, the first three levels were considered a beginner level, and the next three levels were defined as an intermediate level because inversions and twists were involved. A beginner level routine consisted of skills that did not involve inversions; common skills were tuck/pike/straddle jumps and back/seat/front drops. At the intermediate level, skills involved twisting into the drops, such as half twist to back or front drop, and front and back inversions were also performed. The researcher used these progressions as a means to compose the participants' two routines. For example, if the child was in level one, approximately 10 skills would be provided on a progression sheet for the child to achieve before moving up to the next level. Therefore, the research would take these 10 skills, and create two equally difficult five-skill routines. In the case where the child was performing inversions, the researcher ensured the two routines consisted of the same number of flips and rotations.

Once the routines were determined, half the participants performed their FSM routine on the first pre-test day and the other half performed their FSM routine on the second pre-test day. Both pre-test days followed the same procedure. On each pre-test day, the participants were given verbal instructions about the five-skill routine, including the specific order in which the skills were to be performed. Immediately after, the self-efficacy questionnaire and the MOSS were completed. The child then completed four trials of the routine. They were provided with reminders of the skills to be performed if they could not remember the original instructions. After this, the Strategic Planning Questionnaire was administered. Eight more trials of the routine were then performed with the completion of three other questionnaires (one after the eighth trial and two after the twelfth trial) related to other self-regulatory processes. The last trial videotaped was used as their pre-test performance measure. The focus of this article is on physical performance and measures related to the forethought phase. Therefore, the three other questionnaires will not be expanded upon. No FSM video was provided during the pre-test.

\section{Acquisition phase}

Similar to the pre-test, the acquisition days alternated between the FSM routine and the control routine, continuing in the same order as the participant had began at pre-test. Additionally, the focus of the measures on each acquisition day concerned one of Zimmerman's (2000) tri-cyclical phases. That is, the measures were related to those self-regulatory processes and beliefs found in either the forethought, performance control, or self-reflection phases, with the exception of one forethought measure (MOSS) being included in the performance control testing days. We chose to proceed in this manner so as not to overwhelm the participants with too many measures on any particular day and to maintain consistency in the instructions; i.e., think about your thoughts before you began your practice trials. Given that it is possible that effects of the FSM could occur differently depending on the stage of learning, participants were assigned, in a counterbalanced fashion, according to the "testing phase" of the day. Participants rotated through one of these three orders across the six acquisition testing days; (1) Forethought (F), Performance Control (PC), Self-Reflection (SR); (2) SR-F-PC; or (3) PC-SR-F. Given our interest in the forethought phase measures, only the procedures of these sessions will be described (although the other sessions followed virtually the same procedures).

At the beginning of each acquisition day, the participant either viewed the FSM video three times, or heard the verbal description of the routine three times, and then completed the self-efficacy measure, followed by the questions relating to goal setting. A total of 10 practice trials occurred for each session where the last trial was videotaped and used as their performance measure. These 10 trials were divided into two blocks of five trials, with a mid-way viewing of the FSM video three times or the verbal instructions repeated three times. The testing session concluded with the completion of the Strategic Planning Questionnaire. As mentioned, the MOSS questionnaire was given during the performance control sessions. The MOSS was administered immediately after either viewing the FSM video or hearing the instructions, just before the first block of five practice attempts.

\section{Retention test}

Continuing in the alternating order assigned during pre-test, participants completed the retention test $24 \mathrm{~h}$ after the last acquisition session for both the FSM routine and the control routine on the same day. As done in the pre-test, the routine was verbally stated then the self-efficacy and MOSS measures were administered. Four trials of the routine were performed, followed by the Strategic Planning Questionnaire. Eight more trials of the routine were performed, interspersed with the completion of three other self-regulatory measures. No FSM video was provided during the retention test.

\section{DATA ANALYSIS}

Due to absences during the summer camp and video difficulties, data from four participants were discarded. Thus, 27 participants were used in the analysis; 10 participants were in the acquisition day orders of F-PC-SR, 8 were in the order of SR-F-PC, and 9 were in PC-SR-F. This also resulted in 12 participants (Males $n=5$, Females $n=7$ ) with a mean age of 9.72 years learning the control routine on Day 1. Eight of these learned beginner level routines, whereas four were at the intermediate level. The remaining 15 participants 
(Males $=7$, Females $=8$ ) had 9 children at the beginner level and 6 at the intermediate level. The mean age of this group was 10.9 years and on Day 1 they started with the FSM video routine.

Data analysis of the goal setting data involved first determining whether participants established a goal or not. Thereafter, each goal statement was coded to determine whether it was a general goal (e.g., "just do it well") or a specific goal (e.g., "to straighten my legs on the pike jump"). For the remaining measures, prior to conducting any statistical analyses, preliminary analyses confirmed there were no differences between the FSM routine and the control routine for the dependent variables on any of the pre-test scores. It has been recommended that when variables examined are conceptually distinct and/or have been investigated previously using univariate analysis, or the research is exploratory, separate analyses of variance (ANOVAs), rather than multivariate ANOVA should be conducted (Huberty and Morris, 1989). Given this recommendation and the applicability of these factors to our experiment, separate ANOVAs were computed. In addition, because our interest was in the effect of the intervention on base-line performance, and its potential learning effects, we included the pre-test, acquisition, and retention scores in the analysis, a statistical design often used in motor skill acquisition research (e.g., Weiss et al., 1992; Guadagnoli et al., 2002). Finally, because we are using multiple measures within one sample population, Bonferroni adjustment was used (Tabachnick and Fidell, 2001) and $p<0.02$ was set for significance.

Simply relying on statistical significance testing has been argued to limit the understanding and applicability of research findings (Fan, 2001), thus effect size values are reported to complement the significance testing. In this study, effects sizes are reported as partial eta squared $\left(\eta_{\mathrm{p}}^{2}\right)$; a partial eta squared of less than or equal to 0.06 is considered small, while a partial eta squared between 0.06 and 0.14 is medium, and partial eta squared of greater than 0.14 is large (Kinnear and Gray, 2008). In addition to effect sizes, the reporting of confidence intervals has also been advocated (Capraro, 2004). As such, means and their confidence intervals are provided for each of the self-regulatory measures.

\section{RESULTS \\ PHYSICAL PERFORMANCE}

The average of the two judges' scores was used as the physical performance indicator. A 2 (condition) $\times 5$ session (pre-test, 3 intervention sessions, post-test) repeated measures ANOVA, with the Greenhouse-Geisser correction, was done to assess whether there were differences between the average physical performance score across sessions and between conditions. Results indicated that participants showed an improvement trend across sessions $F(2.70$, $70.08)=3.20, p=.033, \eta_{\mathrm{p}}^{2}=0.11$; however, with the Bonferroni corrected alpha at 0.02 , this increase is not significant (see Figure 1). Results also showed a significant difference between conditions $F(1$, 26) $=7.59, p=0.01, \eta_{\mathrm{p}}^{2}=0.23$. Participants scored significantly better on the FSM routine than on their control routine (see Figure 1). No interaction effects were found.

\section{OUESTIONNAIRE DATA}

The same design was used for each of the questionnaires; a 3 Order (F-PC-SR; PC-SR-F; SR-F-PC) $\times 2$ Condition [FSM; control (C) $\times 3$ Session (pre-test; acquisition; post-test) mixed ANOVA with repeated measures on the last two factors, and the GreenhouseGeisser correction. Order of questionnaire administration was not a significant factor for any of the variables measured, consequently Table 1 shows all means and confidence intervals collapsed across order. The analysis of the average scores of the Strategic Planning Questionnaire showed no significant differences across session or across conditions; all $F_{S}<1.0$. As such, participants did not adopt strategic planning differently at their pre-test than at post-test, nor did they strategically plan differently for their FSM routine than for their $\mathrm{C}$ routine. These non-statistical findings were confirmed by small effect sizes.

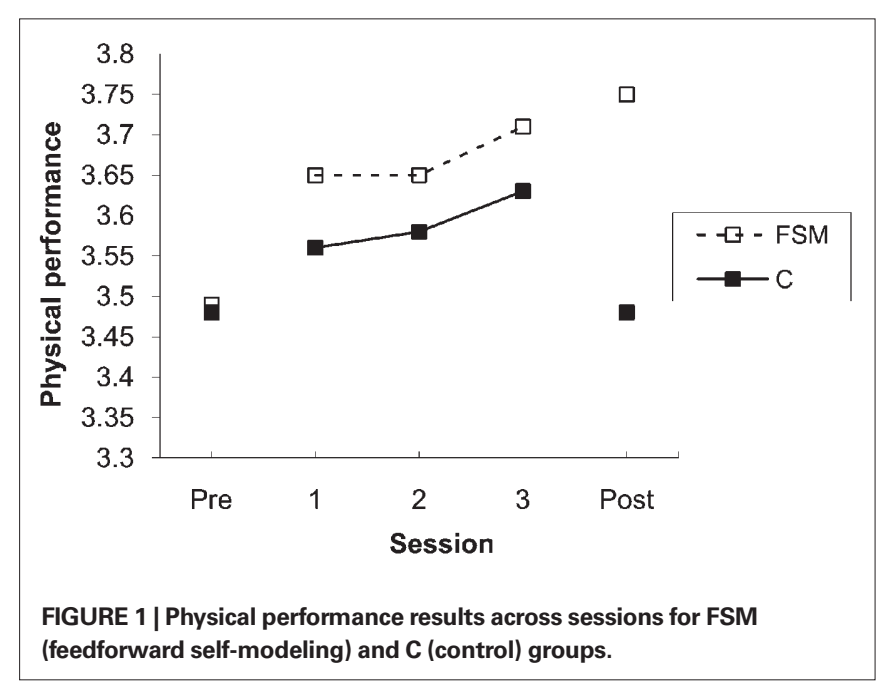

Table 1 | Means and confidence intervals of self-regulatory processes.

\begin{tabular}{|c|c|c|c|}
\hline Session & Intervention & Mean & $95 \% \mathrm{Cl}[]$ \\
\hline \multicolumn{4}{|c|}{ STRATEGIC PLANNING } \\
\hline \multirow[t]{2}{*}{ Pre-test } & SM & 3.10 & {$[2.83,3.34]$} \\
\hline & $\mathrm{C}$ & 3.10 & {$[2.88,3.32]$} \\
\hline \multirow[t]{2}{*}{ Mid-session } & SM & 2.88 & {$[2.54,3.22]$} \\
\hline & $\mathrm{C}$ & 2.93 & {$[2.58,3.28]$} \\
\hline \multirow[t]{2}{*}{ Post-test } & SM & 2.83 & {$[2.46,3.20]$} \\
\hline & $\mathrm{C}$ & 2.93 & {$[2.57,3.29]$} \\
\hline \multicolumn{4}{|c|}{ MOTIVATION } \\
\hline \multirow[t]{2}{*}{ Pre-test } & SM & 15.92 & {$[14.63,17.22]$} \\
\hline & $\mathrm{C}$ & 15.615 & {$[14.38,16.85]$} \\
\hline \multirow[t]{2}{*}{ Mid-session } & SM & 16.23 & {$[14.78,17.69]$} \\
\hline & $\mathrm{C}$ & 16.00 & {$[14.72,17.28]$} \\
\hline \multirow[t]{2}{*}{ Post-test } & SM & 16.27 & {$[14.88,17.66]$} \\
\hline & $\mathrm{C}$ & 16.27 & {$[14.92,17.62]$} \\
\hline \multicolumn{4}{|c|}{ SELF-EFFICACY } \\
\hline \multirow[t]{2}{*}{ Pre-test } & SM & 62.09 & {$[54.53,69.65]$} \\
\hline & C & 60.30 & {$[53.10,67.51]$} \\
\hline \multirow[t]{2}{*}{ Mid-session } & SM & 70.04 & {$[63.15,76.94]$} \\
\hline & $\mathrm{C}$ & 71.70 & {$[64.36,77.85]$} \\
\hline \multirow[t]{2}{*}{ Post-test } & SM & 72.36 & {$[65.67,79.05]$} \\
\hline & $\mathrm{C}$ & 72.64 & {$[64.90,80.38]$} \\
\hline
\end{tabular}

Cl, confidence interval; SM, self-modeling; C, control. 
The total MOSS-challenge sub-scale score was used as a motivation indicator. While the trend for motivation was increasing for both C and FSM conditions, with higher motivation for the FSM group initially (see Table 1), these differences were not found to be significant, all $F$ s $<1.0$. These non-statistical findings were also confirmed by small effect sizes. Self-efficacy scores showed a significant improvement across sessions $F(1.60,41.52)=12.1, p=.00$, $\eta_{\mathrm{p}}^{2}=0.318$. Differences between FSM and C groups, however, were not significant, nor were there any significant interactions. Thus, no difference in participants' sense of self-efficacy for their FSM routine and their $\mathrm{C}$ routine were noted.

\section{GOAL SETTING}

While the participants set more goals for their FSM routine $(37.0 \%)$ than their C routine (27.6\%), the non-parametric McNemar test results indicated that this difference was not significant. Of those participants who set goals, $55 \%$ of the goals set for the FSM routine were specific while only $25 \%$ of goals set for the C routine were specific. These differences, however, were also not significant, as verified by the non-parametric McNemar test.

\section{DISCUSSION}

One of the main objectives of this research was to examine whether a FSM intervention would enhance motor skill acquisition in children learning trampoline skills. The main effect obtained for condition indicated that the routine accompanied by the FSM video was acquired better than the routine that only received verbal instruction. Moreover, the large effect size indicates that the effects are meaningful both statistically and practically. Certainly, in subjective sport, the difference of 0.27 in a final score influences the outcome of the competition greatly. As an example, in the Beijing 2008 Olympics, the value separating the Bronze and Silver medalists in the male category was only 0.10 (Entertainment Sports Programming Network, 2008).

A second main objective involved exploring possible variables that may help to explain why the FSM video enhanced skill acquisition. As mentioned, researchers have argued that benefits of a selfmodeling intervention may arise from enhanced self-regulatory processing (e.g., Schunk, and Hanson, 1989; Starek, and McCullagh, 1999; Winfrey and Weeks, 1993). Our data, however, does not support this thesis as we did not obtain any differences for any of the varied self-regulatory processes and beliefs that were measured. Certainly, there are always limitations to consider with one's measurement tools. For example, the Strategic Planning Questionnaire was a newly developed questionnaire specific to this research. Perhaps further validation and testing of that questionnaire would allow one to more resolutely stand by its findings.

The MOSS-challenge sub-scale, however, has been validated and used with children in the past (Weiss et al., 1985). In comparison to research that is most aligned with ours, Clark and Ste-Marie (2007) reported that children who had watched a positive self-review selfmodeling video were more intrinsically motivated to learn swimming skills than those who did not. As such, we were surprised that our findings did not show the same effects. A key difference between our experiment and Clark and Ste-Marie's lies in the experimental design. In their research, they had separate groups for the use of the self-modeling video versus no video, whereas ours was a within design such that the same group had a self-modeling video for one routine, but not the other. Consequently, it is possible that there was transference to the non-video routine in terms of general factors such as motivation and strategies to incorporate when learning trampoline routines. Thus, future research may want to maintain a between condition design to better isolate the contributions of the self-modeling video.

This argument about transference is more difficult to sustain in relation to the self-efficacy findings, as this measure was very specific to the routine which received the FSM video. That is, learners were only provided with the apparent mastery experience for the FSM routine and not the control routine. Again, one can question the viability and sensitivity of the questionnaires used to measure this psychological variable; however, it was developed using Bandura's (2006) guidelines and the pattern of responses by the participants did reflect that they understood the meaning of the items in the questionnaire. Also of note is that the measure did show that learners were attaining higher levels of self-efficacy throughout the learning experience, thus the measure was sensitive to these changes. The lack of a difference between the two groups suggests that self-efficacy is not a critical factor. This finding is similar to others who have also predicted higher levels of self-efficacy as a result of a self-modeling intervention, but not obtained such results (Starek and McCullagh, 1999; Winfrey and Weeks, 1993; Ram and McCullagh, 2003; Clark and Ste-Marie, 2007). Therefore, while the logic that learners would perform better due to higher levels of self-efficacy obtained from viewing a successful performance corresponds well with Social Cognitive Theory, self-efficacy does not have strong support as an explanatory variable for self-modeling benefits to date.

The lack of findings with our self-regulatory measures obviously leads one to question what the root cause of these benefits may be. While we do not want to exclude self-regulation completely, it may be time to examine other informational processes that may be implicated. Bandura (1986) does have attention to relevant cues and their retention in a cognitive representation as two key factors associated with the observational learning process. The FSM video may be serving to provide a better cognitive representation of the skills as a result of observing critical features in the skill. In line with Deprati et al.'s (2007) proposal, viewing the self may provide the learner with information concerning subtle aspects of the movement to facilitate learning. The goal setting data, while not significant, does suggest that the self-modeling video focused the learners on specific details of the trampoline performance. For example, one participant indicated that they wanted "to straighten my legs on the pike jump" (P15). One can assume that observing the video enabled them to determine the changes in performance needed to improve the routine. Of interest is that this participant's comment also shows that while the self-modeling video showed a higher level performance than the participant could obtain, it was still not a "perfect" performance. Disentangling participants' observations of what still needs improvement from those that are correctly performed would be an interesting avenue in future research determining the benefits of self-modeling over other modeling interventions.

Another line of thought comes from research that has attributed positive affect to enhancing motor learning (e.g., Lewthwaite and Wulf, 2009). For example, research on self-control has shown that 
learners prefer to receive feedback following more successful performance trials than unsuccessful performance trials (Chiviacowsky and Wulf, 2002). Further research by this team also showed that feedback provided after successful trials enhanced motor learning over those provided after unsuccessful trials (Chiviacowsky and Wulf, 2007). Using a social-comparative research design, Lewthwaite and Wulf (2009) have also showed that motor skill acquisition is enhanced when learners believe they are performing better than the norm. These lines of research argue for motor learning advantages that may be related to important psychological variables like positive affect, similar to that which would be expected from watching one self perform successfully. Thus, in keeping with the statement that self-regulation variables should

\section{REFERENCES}

Bandura, A. (1986). Social Foundations of Thought and Action: A Social Cognitive Theory. Englewood Cliffs, NJ: Prentice-Hall.

Bandura, A. (1997). Self-Efficacy: The Exercise of Control. New York, NY: Freeman.

Bandura, A. J. (2006). "Guide for constructing self-efficacy scales," in SelfEfficacy Beliefs of Adolescents, eds F. Pajares and T. Urdan (Greenwich, CT: Information Age Publishing, Inc.), 307-338.

Capraro, R. M. (2004). Statistical significance, effect size reporting, and confidence intervals: best reporting strategies. J. Res. Math. Educ. 35, 57-62.

Chiviacowsky, S., and Wulf, G. (2002). Self-controlled feedback: does it enhance learning because learners get feedback when they need it? Res. Q. Exerc. Sport 73, 408-415.

Chiviacowsky, S., and Wulf, G. (2007). Feedback after good trials enhances learning. Res. Q. Exerc. Sport78, 40-47.

Clark, S. E., and Ste-Marie, D. M. (2007). The impact of self-as-a-model interventions children's self regulation of learning and swimming performance. J. Sports Sci. 25, 557-586.

Deci, E. L. (1975). Intrinsic Motivation. New York, NY: Plenum Press.

Deprati, E., Wreissenegger, S., and Lacquaniti, F. (2007). Knowledge of one's kinematics improves perceptual discrimination. Conscious. Cogn. 16, 178-188.

Dowrick, P. W. (1999). A review of self modeling and related inventions. Appl. Prev. Psychol. 8, 23-39.

Dowrick, P. W., and Dove, C. (1980). The use of self-modeling to improve the performance of spina bifida children. J. Sports Sci. 13, 51-56.

Entertainment Sports Programming Network. (2008). 2008 Summer
Olympics Results - Trampoline. Available at: http://sports. espn.go.com/oly/summer/08/ results?discId $=23$

Fan, X. (2001). Statistical significance and effect size in education research: two sides of a coin. J. Educ. Res. 94, 275-282.

Feltz, D. L., Short, S. E., and Sullivan, P. J. (2008). Self-Efficacy in Sport: Research and strategies for Working with Athletes, Teams, and Coaches. Champaign, IL: Human Kinetics.

Guadagnoli, M., Holcomb, W., and Davis, M. (2002). The efficacy of video feedback for learning the golf swing. $J$. Sports Sci. 20, 615-622.

Holmes, P., and Calmels, C. (2008). A neuroscientific review of imagery and observation use in sport. J. Mot. Behav. 40, 433-445.

Huberty, C. J., and Morris, J. D. (1989). Multivariate analysis versus multiple univariate analysis. Psychol. Bull. 105, 302-308.

Kinnear, P. R., and Gray, C. D. (2008). SPSS 15: Made Simple. New York, NY: Psychology Press.

Kyllo, L. B., and Landers, D. M. (1995). Goal setting in sport and exercise: a research synthesis to resolve the controversy. J. Sport Exerc. Psychol. 17, 117-137.

Law, B., and Ste-Marie, D. M. (2005). Effects of self-modeling on figure skating jump performance and psychological variables. Eur. J. Sport Sci. 5, 143-152.

Lewthwaite, R., and Wulf, G. (2009). Social-comparative feedback affects motor learning. Q. J. Exp. Psychol. 63, 738-749.

Locke, E. A., and Latham, G. P. (1990). Performance. Englewood Cliffs, NJ: Prentice Hall.

Murphy, K. R., and Davidshofer, C. O. (2005). Psychological Testing: Principles A Theory of Goal Setting and Task

not be excluded, there is still reason to examine the psychological influences of positive affect and its impact on motor skill learning in the context of self-modeling benefits.

In conclusion, children acquired a trampoline routine better when provided with a FSM video than just receiving verbal instructions during the acquisition phase. The learning advantage gained by the self-modeling intervention, however, cannot be explained by the self-regulatory belief of self-efficacy, or the processes of goal setting, intrinsic motivation, and strategic planning as measured herein. Future research on the topic of self-modeling should perhaps consider other informational processes gained from a FSM video, while still examining psychological variables associated with learning conditions that create positive affect.

and Applications, 6th Edn. Upper Saddle River, NJ: Pearson Education, Inc.

Ram, N., and McCullagh, P. (2003). Selfmodeling: influence on psychological responses and physical performance. Sport Psychol. 17, 220-241.

Rymal, A. M., Martini, R., and Ste-Marie, D.M. (2010). Self-regulatory processes employed during self-modeling: a qualitative analysis. Sport Psychol. 24, $1-15$.

Schmidt, R.A., and Wrisberg, C.A. (2008) Motor Learning and Performance, 4th Edn. Champaign, IL: Human Kinetics. Schunk, D. (1987). Peer models and children's behavioural change. Rev. Educ. Res. 57, 149-174.

Schunk, D. H., and Hanson, A. R. (1989) Self-modeling and children's cognitive skill learning. J. Educ. Psychol. 81, 155-163.

Starek, J., and McCullagh, P. (1999). The effect of self-modeling on the performance of beginner swimmers. Sport Psychol. 13, 269-287.

Tabachnick, B. G., and Fidell, L. S. (2001). Using Multivariate Statistics. Needham Heights, MA: Allyn and Bacon.

Urbina, S. (2004). Essentials of Psychological Testing. Hoboken, NJ: John Wiley and Sons, Inc.

Weinberg, R. S., and Butt, J. (2005). "Goal setting in sport and exercise domains: the theory and practice of effective goal setting," in Handbook of Research in Applied Sport and Exercise Psychology: International Perspectives, eds D. Hackfort, J. L. Duda, and R. Lidor. (Morgantown, WV: West Virginia University Press), 129-144.

Weiss, M., Ebbeck, V., and Rose, D. (1992) "Show and tell" in the gymnasium revisited: developmental differences in modeling and verbal rehearsal effects on motor skill learning and performance. Res. Q. Exerc. Sport 63, 292-301.
Weiss, M. R., Bredemeier, B. J., and Shewchuk, R. M. (1985). An intrinsic/ extrinsic motivation scale for the youth sport setting: a confirmatory factor analysis. J. Sport Psychol. 7, 75-91.

Winfrey, M. L., and Weeks, D. L. (1993). Effects of self-modeling on selfefficacy and balance beam performance. Percept. Mot. Skills77,907-913.

Zimmerman, B. J. (2000). “Attaining selfregulation: a social cognitive perspective," in Handbook of Self-regulation, eds M. Boekarts, P. R. Pintrich, and M.Zeidner (San Diego, CA: Academic Press), 13-39.

Zimmerman, B. J. (2008). Investigating self-regulation and motivation: historical background, methodological developments, and future perspectives. Am. Educ. Res. J. 45, 166-183.

Conflict of Interest Statement: The authors declare that the research was conducted in the absence of any commercial or financial relationships that could be construed as a potential conflict of interest.

Received: 28 April 2011; paper pending published: 25 May 2011; accepted: 22 June 2011; published online: 07 July 2011.

Citation: Ste-Marie DM, Vertes K, Rymal $A M$ and Martini R (2011) Feedforward self-modeling enhances skill acquisition in children learning trampoline skills. Front. Psychology 2:155. doi: 10.3389/ fpsyg.2011.00155

This article was submitted to Frontiers in Movement Science and Sport Psychology, a specialty of Frontiers in Psychology. Copyright $\odot 2011$ Ste-Marie, Vertes, Rymal and Martini. This is an open-access article subject to a non-exclusive license between the authors and Frontiers Media SA, which permits use, distribution and reproduction in other forums, provided the original authors and source are credited and other Frontiers conditions are complied with. 